\title{
INTEGRATION OF PATIENT SPECIFIC MRI IMAGING DATA INTO A STOCHASTIC LOW-GRADE GLIOMA MODEL
}

\author{
Thaís Roque ${ }^{1}$, Maria Concepcion Garcia Otaduy ${ }^{2}$ and Waldemar Zylka ${ }^{1}$ \\ ${ }^{1}$ Westfälische Hochschule Gelsenkirchen, Germany, ${ }^{2}$ University of São Paulo, Brazil \\ thasolque@googlemail.com
}

\begin{abstract}
A method to validate a previously developed multi-scale stochastic tumor model using patient specific quantitative MRI images is developed. For a specific clinical low-grade glioma case, model parameters are extracted from MRI, an extrapolation of the spatio-temporal evolution is computed and the result compared to the actual tumor development available in another MRI image. The model describes qualitatively and quantitatively avascular tumor growth and its typical structure. The growth rate (radial velocity) of the computed tumor is found to be constant as it is in the clinical case. However, growth rate values differ significantly.
\end{abstract}

Keywords: patient specific, validation, tumor modeling, low-grade glioma, active cellular automaton

\section{Introduction}

Modeling of tumor growth has become a powerful tool to predict tumor spatio-temporal evolution and its response to therapy. For the case of low-grade glioma, the purpose of this study was to validate a previously developed multiscale stochastic model for avascular tumor growth which includes cell-cycle regulation [1]. This model represents an active cellular automaton allowing for proliferation and spatial spread of tumor cells. In addition, the model accounts for oxygen distribution and various fractionation schemes of external radiotherapy [2].

The validation of the tumor growth model was performed retrospectively using longitudinal time series of Magnetic Resonance Images (MRI) of one clinical low-grade glioma case. A methodology was developed to extract patient specific values for model parameters from MRI images at two subsequent time points. The numerical simulations were run with these parameters to predict the spatio-temporal tumor evolution at a third point in time. These predictions are than compared to patients MRI acquired at the same time.

\section{Methods}

The model [1, 2] accounts for biological cells that are in proliferating (G1, S, G2 and mitosis) and quiescent phases (G0). Cells change phases with certain probabilities depending on oxygen level. For insufficient oxygen partial pressure, $p \mathrm{O}_{2}$, the cell becomes quiescent, but can reenter the cycle within a time interval in case conditions change to normoxic. In case of chronic hypoxia the cell becomes necrotic and is removed from cell cycle after a lysis time. The model is able to simulate tumor response to radiotherapy using the linear-quadratic (LQ) and the linearquadratic-linear (LQ-L) approaches[2].

Cells survive, divide or die with given probabilities, depending on their current state and on their oxygen level. The proliferation is driven by a Gompertz law which is influenced by the oxygen level. For the brain tissue $\mathrm{pO}_{2}$ range is $\mathrm{pO}_{2}=25-50 \mathrm{~mm} \mathrm{Hg}$ [3]. If the number of biological cells reaches the carrying capacity, i.e. the maximum number of biological cells that a lattice cell can sustain, they are shifted to the edge because of oxygen supply [1]. This biological cell relocation is governed by stochastic approaches and models a diffusive spherical spread of the tumor.

The multi-scale formulation corresponds to an active cellular automaton approach. It is discretized with $100 \mathrm{x}$ 100 lattice cells on a two-dimensional domain. A Mooreneighborhood is implemented. Transition rules for biological cell relocation on the lattice are non-homogenous and non-local. Proliferation and spatial diffusive spread of biological cells are implemented during avascular tumor growth, i.e. low-grade gliomas, which are described by the diffusion constant $D$ and the net proliferation rate $\rho$. The carrying capacity of a lattice cell is $k=11,304$ biological cells with diameter $d=13 \mu \mathrm{m}$ [4]. Low-grade gliomas are initially slowly evolving, but can change the grade and invade other areas of the brain. Their development is linear in time [5]. The total number of glioma cells may double exponentially, but isolated glioma cells infiltrate to other regions of the brain resulting in a constant radial velocity for tumor bulk growth [5].

As an example this paper presents a retrospective investigation of a 52-year-old male patient treated at the Hospital das Clínicas da Universidade de São Paulo. The patient underwent brain MRI examination including pre- and postGd T1-weighted in several acquisition planes, FLAIR, T2and T2*-weighted, perfusion and DWI acquisitions. The initial cell number $N_{0}$ is estimated from DWI images of the patient [4]. Diffusion and prolifaration parameters, $D$ and $\rho$, are calculated using specific patient data, such as tumor volume and diameters, extracted from T1- and T2weighted MRI images [5, 6]. With the Fisher's approximation, $v=2 \sqrt{D \rho}$, the radial velocity of the tumor bulk $v$ can be calculated. The invisibility index, $I=D / \rho=V_{i} / V_{t}$, refers to the fact that the size of gliomas is known to be not entirely detectable in MRI. $V_{i}$ and $V_{t}$ represent the infiltrative volume (visible in the T2-image) and the solid tumor volume (visible in the T1-image), respectively [6]. Once $v$, $V_{i}$ and $V_{t}$ are obtained from MRI, the parameters $D$ and $\rho$ of the automaton are known for every specific patient. 


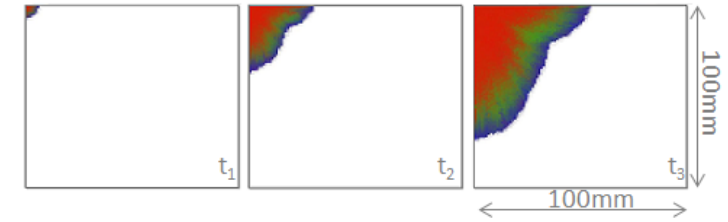

Figure 1: Spatio-temporal tumor evolution in one quadrant.

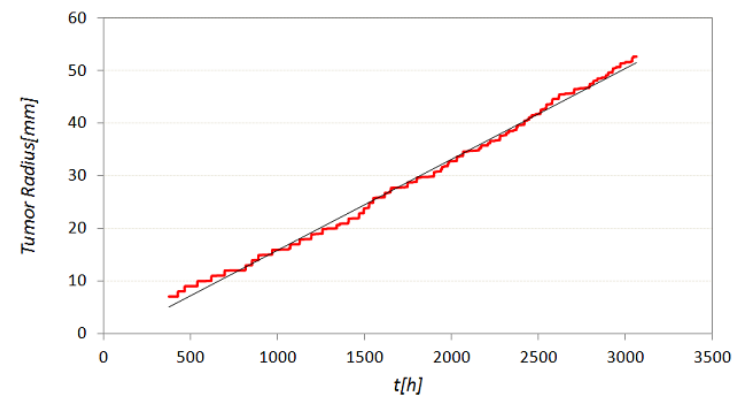

Figure 2: Time evolution of the averaged tumor radius.

\section{Results}

The initial simulation parameters, i.e. $t_{0}=0 d$, were: $N_{0}=11,200, D=41.04 \mathrm{~mm}^{2} / y, \rho=11.01 y^{-1}$ and $p \mathrm{O}_{2}=32.5 \mathrm{mmHg}$. Figure 1 shows the results of the simulated tumor: a necrotic core (red) is surrounded by two rims of quiescent (green) and proliferating (blue) biological tumor cells. Empty lattice cells appear transparent (white). The simulated tumor radius was of $r_{1}=6.99 \mathrm{~mm}$ for the time point $t_{1}=16 d$ (left-hand side), $r_{2}=29.78 \mathrm{~mm}$ for $t_{2}=78 d$ (middle) and $r_{3}=52.67 \mathrm{~mm}$ for $t_{3}=128 \mathrm{~d}$. Figure 2 displays the time evolution between $t_{1}$ and $t_{3}$ of the simulated tumor bulk radius which fits a linear growth given by $r(t)=0.0172 t-1.3125$. Thus, the growth rate, i.e. radial velocity, from simulations in this clinical case is $v=0.0172 \mathrm{~mm} / \mathrm{h}=150.67 \mathrm{~mm} / \mathrm{y}$. Because the patient underwent three sessions of external radiotherapy in the 123.,124. and 125. day, in a second simulation external radiation was delivered at these days once a day with a dose $d=1.8 G y$. As a result, the simulated tumor radius at $t_{3}$ was $r_{3}=50.13 \mathrm{~mm}$ and the total number of cells was $N=22,040,900$. In case of the non-irradiated tumor simulation the cell number is $N=24,062,701$.

\section{Discussion and Conclusions}

This paper presents the validation of an active cellular automaton model using quantitative MRI patient data and the predictions of tumor spatio-temporal evolution of this model for a clinical case. Patient data obtained from T1-, T2 and DWI MRI images were converted into quantitative parameters used as input in the computational calculations. Low-grade glioma bulk develops linear in time [5] and (as long as avascular) it consists of a necrotic core surrounded by two rims of quiescent and proliferating cells. Figures 1 and 2 demonstrate this structure generated by the automaton model for the specific patient data.

While the tumor structure is qualitatively well represented, the values of tumor radial velocities differ between simulations and clinical data. The simulated radial (growth) velocity $v=150.67 \mathrm{~mm} / y$ is larger than the average velocity obtained from the tumor bulk evolution found in T1weighted MRI $\bar{v}=(32.41 \pm 4.31) \mathrm{mm} / y$. The simulated tumor radius was not significantly affected by the first three sessions of radiotherapy, which is in agreement with clinical observations [7]. Nevertheless, the overall simulated tumor radius is by $151.05 \%$ larger than the radius deduced from T1-weigthed MRI image at $t_{3}$. This might be due to a too long time interval used for extrapolation to $t_{3}$.

In the brain structures like white matter myelin fibers, that act as barriers to the free diffusion of particles, are present. Prospectively, they will be integrated in the model based on Diffusion Tensor MRI, which calculate water diffusion anisotropy caused by the presence of white matter fibers. This investigation is of crucial importance as gliomas preferentially migrate along the direction of these fibers. In addition, inclusion of data from Positron Emission Tomography, which is able to quantitatively measure the absolute oxygen value, is planned.

\section{Acknowledgement}

We acknowledge Fábio Diniz collection of patient data.

\section{Bibliography}

[1] N. Rudigkeit et al., "Modeling tumor growth and treatment using imaging data," Biomedical Engineering, vol. 56, no. 1, p. 422, 2011.

[2] T. Roque et al., "Biomedical effectiveness in hypofractionation: Modelling tumor survival probability for large doses with a stochastic cell-cycle model," Biomedical Engineering, vol. 57, no. 1, pp. 202-205, 2012.

[3] E. Wilensky et al., "Monitoring brain tissue oxygenation after severe brain injury," Nursing, vol. 35, no. 2, pp. 1-4, 2005.

[4] N. Atuegwu et al., "The integration of quantitative multi-modality imaging data into mathematical models of tumors," Phys. Med. Biol., vol. 55, pp. 2429-2449, 2010 .

[5] S. Jbabdi et al., "Simulation of anisotropic growth of low-grade gliomas using diffusion tensor imaging," Magnetic Resonance in Medicine, vol. 54, no. 3, pp. 616-624, 2005.

[6] C. Suarez et al., "Mathematical modeling of human glioma growth based on brain topological structures: Study of two clinical cases," Plos One, vol. 7, no. 6, pp. 1-11, 2012.

[7] D. Brandsma et al., "Clinical features, mechanisms, and management of pseudoprogression in malignant gliomas," Seminars in radiation oncology, vol. 9, no. 5, pp. 453-461, 2008. 